\title{
Writing programs on the Apple for the student psychology laboratory: Routines, subroutines, and sources of information
}

\author{
DOUGLAS B. EAMON \\ University of Wisconsin-Whitewater, Whitewater, Wisconsin \\ and \\ DARRELL L. BUTLER \\ Ball State University, Muncie, Indiana
}

\begin{abstract}
Writing programs for the student psychology laboratory on the Apple II series of microcomputers presents the programmer with a number of problems. Among these are increasing program execution speed, obtaining precise response and interval timing, reducing program memory requirements, obtaining efficient disk $\mathrm{I} / \mathrm{O}$, providing good error trapping, and presenting clear screen displays. Since Applesoft BASIC is not equipped with many of the desirable features of a more advanced language, ways to solve these problems are not obvious. This paper suggests a number of potential solutions and provides additional sources of information.
\end{abstract}

In this paper we present a number of observations regarding the development of programs for use in the student laboratory. Rather than provide an overview of the entire process, we focus on some specific problems we have encountered and how we solved or, in some cases, circumvented them (see Perera, 1987, for useful advice and many practical details on publication). Many of our solutions are in the form of tools or utilities that have wide application. These include programming aids, routines and subroutines that are easily incorporated into a variety of types of programs, and some hardware accessories that are especially useful. This paper is directed to the novice or intermediate programmer rather than the proficient or professional, who is already aware of these and many other techniques.

Since we program almost entirely on the Apple and compatible series of microcomputers, these remarks pertain largely to that machine and its peculiarities. Information about the Apple IIgs is meager at the time of this writing; however, we believe that the procedures discussed here will work on the Apple IIg systems.

\section{THE APPLE II SERIES AND COMPATIBLES}

The Apple II has been around since about 1977. Compared with many of its currently available competitors, it is underpowered, overpriced, poorly designed, and generally obsolete in terms of native language (Applesoft

\footnotetext{
Douglas B. Eamon's mailing address is: Department of Psychology, University of Wisconsin-Whitewater, Whitewater, WI 53190. Darrell L. Butler's mailing address is: Department of Psychology, Ball State University, Muncie, IN 47306.
}

BASIC) and hardware. Nonetheless, it remains the most popular computer used in instructional applications, including those in the student psychology laboratory (Eamon \& Butler, 1985). The result is that a great many hardware and software tools have become available for programmers using the Apple; many of these are designed to overcome the Apple's shortcomings and to extend its strengths. In addition, so many individuals have been faced with the problems inherent in programming for the Apple that a substantial literature has become available providing solutions (or partial solutions) for many problems. One of the primary purposes of this paper is to attempt to categorize commonly encountered problems associated with using the Apple, and to assemble a list of sources of information on how to deal with them.

\section{TYPES OF APPLICATIONS}

Eamon and Butler (1985), following Castellan (1983), identified a number of applications of computers in the student psychology laboratory. Among these are data generation (generating artificial data based on studententered descriptions of experiments), experiment simulation (replication or near-replication of classic or other experimental studies), and experiment generation (programs that allow students to create new experiments). Although each of these applications presents special problems for the program writer, they often share one or more of the following features: program execution speed is important; precise response or interval timing may be required; programs can be quite large, and often tax the limits of the RAM available for program and data storage; programs frequently need to read and/or write 
to the disk (with some speed); error trapping is critical and must be virtually foolproof; and screen displays must be clean and easy to read (ideally with upper/lowercase). In addition, there are other miscellaneous problems (random permutation, sorting, tone generation, etc.). These are not independent of each other. For example, easily readable screen displays and good error trapping require additional programming lines and, thus, more program RAM and additional execution time. Finally, all of these are exacerbated by Applesoft itself, which is a relatively poor programming environment.

\section{PROBLEMS AND SOME POSSIBLE SOLUTIONS}

Several of the problems we have encountered in Applesoft programming have partial solutions in programming technique, software utilities, and/or hardware. Where appropriate, we have considered these separately.

\section{Program Execution Speed}

The 6502 series of microprocessors, operating at a little over $1 \mathrm{MHz}$, would probably be sufficient for most applications were Applesoft BASIC not an interpreted language. But it is, and many programs could be enhanced by having increased execution speeds. There are a variety of ways in which speeds can be increased. Some of these are well known but worth repeating.

Programming technique. The first three of these are given in the Applesoft II BASIC Programming Reference Manual (Apple Computer, 1978).

1. Variables should be used instead of constants. The value of a variable can be recovered faster than a constant can be converted to its floating point value. The Reference Manual states that this is the "most important speed hint by a factor of 10" (p. 120) (capitals deleted).

2. Frequently used or speed-critical variables should be defined early in the program. Applesoft searches the variable table from the beginning to locate the value of a variable; those encountered (defined) first will be most quickly recovered.

3. Frequently used subroutines or time-critical subroutines should be placed at the beginning of the program. When it encounters a GOTO or GOSUB, Applesoft scans the entire program from the first line until it finds the appropriate line number; those found first are executed more quickly. (The Reference Manual also suggests that the index variable on a NEXT statement may be dropped. We do not recommend this practice, since the minimal increase in speed that might be obtained is usually not enough to overcome the difficulty in reading and editing the program later.)

4. Users cannot read material as fast as it can be printed to the screen. The extra time taken for the user to read instructions or other program output can often be put to use. For example, after printing a screen of text, various functions (e.g., sorting, garbage collection, READing arrays into memory, INPUTting text or data from disk, etc.) can be performed while the user is reading the screen, before returning control to the individual.
5. Output to printers is usually slow relative to program execution. Again, the programmer can take advantage of this time by performing computations at the time the printing is taking place.

6. Sorting and manipulating strings should be avoided whenever possible. If necessary, a procedure that sorts a numeric array whose elements are pointers to the appropriate string elements is faster than moving strings themselves and also avoids garbage collection. A description of this technique can be found in Connolly (1981) or Mitchell (1982); a machine language version (with a quicksort) is also available (Irwin, 1982).

7. In general, users are much more tolerant of waiting a considerable time while something is happening slowly than waiting even short periods when nothing is happening at all. Displaying information about the progress of time-consuming routines is very helpful in alleviating fears that the computer may have "crashed."

Software utilities. 1. There are a variety of machine language routines that provide fast performance of a number of common (and not so common) tasks. These can save a great deal of time and effort, provided they can be adopted for distribution in your programs. Routines are available for a fast simulation of Applesoft's missing PRINT USING command (Bongers, 1982a; Morris, 1980), a fast GOTO processor (Waddell, 1984), fast sorting (Bongers, 1983), fast read/write of text files (Reynolds, 1983), fast garbage collection (Basham, 1985), and so on.

Many machine language routines use the Apple's ampersand (\&) jump vector to interface with BASIC. This can cause problems, since the routines often assume that the ampersand vector will be used exclusively for the particular function; if a program uses several ampersand routines, a procedure is needed to interpret which routine is needed. Ampersand interpreters are provided by Mottola (1981a) and Irwin (1983). Blankenship BASIC (Blankenship, 1984), discussed below under "Getting Around BASIC as a Language," is an extensive set of routines that also allows easy incorporation of additional ampersand routines.

2. An alternative approach to improving program speed is to compile the program. Unfortunately, the increase in speed is not as great as one would like (around 2-3 times), and compilers expand the size of the program (about 1.5 times is typical). Some compilers allow compilation of segments or subroutines of programs; these can then be CALLed from BASIC. An especially good one is ZBASIC (Gariepy, Overton, \& Terry, 1985), and we have also heard good things about Micol BASIC (Micol Systems, 1985).

Hardware. There are several accelerator cards available (e.g., Titan Technologies and Applied Engineering) that can be installed in the Apple. These operate by taking over processing from the 6502. Speed increases are said to be in the range of 2.5-3.6 times. Unfortunately, one cannot assume that the end user of a program will have such a peripheral available. Nevertheless, such a card is a valuable aid to programming and debugging. 


\section{Response and Interval Timing}

Programming technique. For a variety of reasons, counting loops in BASIC is not a satisfactory way to achieve response and interval timing on the Apple. We think the best solution to this problem is to learn assembly language and use an assembler to write the routines needed. Unfortunately, assembly language is difficult to master, and many of us simply do not have the time or the inclination to do so. The Visible Computer: 6502 (Software Masters, 1984a) is an instructive program for learning, provided one can tolerate the condescending and "cute" tone of the accompanying text (Software Masters, 1984b). Other good introductions to assembly language for the Apple are given by Wagner (1982a), Findley (1979), and De Jong (1982). A particularly easy-to-use assembler is The Assembler System (Floeter \& Floeter, 1983c), which is augmented by a collection of Applesoftlike macros called MacroSoft (Floeter \& Floeter, 1983d), including DIM, LET, INPUT, PRINT, IF-THEN-ELSE, REPEAT-UNTIL, etc. Valuable information on interfacing machine language routines with BASIC is given by Devine (1986), Mottola (1981b), Wagner (1982b, 1982c), and Zimmerman (1986). An assembly language reference manual for the 6502 was written by Leventhal (1979).

Several published routines provide timing functions (e.g., Price, 1979, 1986).

Hardware. 1. Synchronizing the screen display with the timing routines is necessary for time-critical applications. On the Apple II+, this can be accomplished using what has come to be called the "Reed modifications" (Reed, 1979), simple hardware modifications to sense the video sync signal and to blank the screen. Gordon and Field (1985) noted that video sync can be done in software on the Apple Ile computer and that hardware changes in the Ile have made Reed's screen-blanking modification obsolete, so they have provided an alternative way of accomplishing this for the Ile. These procedures should be used with machine language routines for presentation and timing. Unfortunately, one cannot assume that end users will have made the appropriate changes.

2. Several companies (e.g., Applied Engineering) manufacture hardware clocks for the Apple, most of which are capable of 1-msec resolution. However, one cannot assume that end users have these clock cards.

3. One of the best and most useful peripheral cards available for the Apple is the 6522 parallel interface card. This is an $\mathrm{I} / \mathrm{O}$ device containing two independently running timers that can be set to time intervals or to wait for a keypress (or other detectable event). It should also be used in conjunction with machine language routines to start it, stop it, and read the time. An excellent description of the use of the 6522 for psychologists can be found in Mueller, Gruenenfelder, Robertson, and Carrell (1982). More detailed descriptions may be found in De Jong (1982) and Leventhal (1979).

\section{Program Size}

With DOS 3.3 installed in its normal location in memory, there are only about 36,000 bytes of RAM avail- able (in a 48K Apple) for storage of both the program and the variables associated with it. If graphics displays are used, available RAM is reduced by about 8,000 bytes per graphics page. The worst case, but not atypical, scenario (with both graphics pages used) reduces available RAM to approximately 20,000 bytes. Although this memory may be adequate for many applications, it is easy to run over that limit, particularly if a program requires many strings or complex variable arrays. We feel that many techniques to reduce program size (including most mentioned here) should be avoided if possible. Nonetheless, there may be situations when every extra byte counts. Some of the following solutions may help.

Programming technique. 1. The best solution to the problem of running out of RAM is to segment the programs; that is, write smaller programs that call each other or a main menu. This has the additional beneficial effect of forcing a minimal amount of structure onto the programs. If variables need to be saved, they can be stored on disk and then read back by the segments that use them. Bongers (1982b) provides a fast and efficient procedure for storing selected arrays, or the program CHAIN (Apple Computer, 1980b) can be used to preserve all of them in memory.

2. If large numeric arrays are necessary, integer variables can achieve a considerable savings (integer variables require only 2 bytes, as opposed to real variables, which require 5 bytes each). There is, however, some loss of speed in the use of integer variables.

3. Another solution may be to INPUT data (e.g., instructions, stimuli, lengthy arrays, etc.) from text files stored on disk, rather than to define them in program lines or to include them as DATA statements within the program. If time is not critical, an entire text page can be stored to disk with the command

\section{BSAVE FILENAME,A $\$ 400, \mathrm{~L} \$ 3 F F$}

and read back in during program execution using

\section{BLOAD FILENAME}

Care should be taken, however, to place the cursor appropriately before reading text pages from disk.

4. Boolean variables should be used when appropriate. For example, many programs treat a Boolean variable as a real variable, as in

$$
\text { IF } \mathrm{X}<>1 \text { THEN } \ldots
$$

when

\section{IF NOT X THEN ...}

saves bytes and executes faster.

An interesting use of Boolean variables is in the "toggle" switch: $X=X=0$. Whenever this statement is encountered, the value of $X$ will change from 0 to 1 , or vice versa.

5. Some punctuation marks can be left out of PRINT statements. Although we do not recommend this as good programming practice, it can save a few bytes. For example, 
PRINT SPC( $\mathbf{N}<10) \mathbf{N}$ “” ”I $\$(N)$

$\operatorname{SPC}(X-\operatorname{LEN}(\mathbf{I}(\mathrm{N}))) \mathrm{D}(\mathrm{N})$

takes five fewer bytes and executes faster than

PRINT SPC( N < 10$)$; N; “ ”; I\$( N); SPC( $X$ - LEN( I\$( N ))); D(N)

6. Assignment of values to strings can often be done using the MID\$( statement. For example,

$$
\begin{aligned}
& \text { IF } X=1 \text { THEN } X \$=\text { "CATS" } \\
& \text { IF } X=2 \text { THEN } X \$=\text { "DOGS" } \\
& \text { IF } X=3 \text { THEN X } \$=\text { "FLIES" } \\
& \text { IF } X=4 \text { THEN X } \$=\text { "ANTS" } \\
& \text { IF } X=5 \text { THEN X } \$=\text { "BEES" }
\end{aligned}
$$

uses more space and executes more slowly than

IF $\mathrm{X}$ THEN $\mathrm{X} \$=$ MID\$(“CATS DOGS FLIES

$$
\text { ANTS BEES ", } 5 * X-4,5 \text { ). }
$$

7. Using multiple statements on each program line also may help. Each line number requires 5 bytes. Be cautious with this one, however, since it is easy to overdo it, and editing these lines becomes more difficult with every extra statement. An alternative solution is to keep the statements on separate lines whenever possible, and then use a program to concatenate them (see \#2 under "Software utilities" below).

8. Upon booting, Apple DOS 3.3 reserves 1,785 bytes of RAM as input buffers for disk $\mathrm{I} / \mathrm{O}$. This is enough to allow three files to be open at the same time. If a program needs less than three files open, the user can recover 595 bytes per file of this space by reducing the number of buffers using the MAXFILES command (e.g., PRINT CHR\$(4) "MAXFILES1"'). This should be done early in the program (before any strings are defined), since MAXFILES resets the HIMEM pointer. This command is not available in ProDOS.

9. DOS 3.3 should be used rather than ProDOS. Although ProDOS is a considerably more advanced disk I/O system, it also occupies more RAM. (It uses all of the space that DOS 3.3 does, plus the $16 \mathrm{~K}$ built-in RAM in the IIe and IIc.) See \#3 under "Software utilities" below.

Software utilities. 1 . There are several programs that remove REM statements from programs (e.g., Apple Computer, 1980a; Floeter, 1981). If your program is heavily REMed (as it should be), it is possible to save a large amount of memory by removing these statements. Of course, it is essential to keep a copy that contains all of the REMs (even if it will not execute).

2. Other programs are available (e.g., Floeter \& Floeter, 1983a) that collapse as many statements as possible onto a single line number and remove those left empty, or shorten variable names (e.g., Floeter \& Floeter, 1983b). Each line number removed saves 5 bytes.
3. Utilities are available (e.g., Basham, 1982) that move DOS 3.3 (but not ProDOS) into the upper $16 \mathrm{~K}$ of memory. The Apple IIe and IIc come with this extra memory built in; it is probably safe to assume that most II+ users have it as well. This releases about 10,000 bytes of RAM, and under many circumstances may be the best solution available.

Hardware. Since the Apple's 6502 can only access $64 \mathrm{~K}$ ( 65,536 bytes), that technically sets the limit on total available memory. Several companies, however, have developed RAM expansion cards that allow memory to be expanded by $128 \mathrm{~K}$ (e.g. , Titan Technologies), 1 MB (e.g., Legend Industries), and even up to $16 \mathrm{MB}$ (Applied Engineering). Unfortunately, these are difficult to use from BASIC unless appropriate software is available, which is almost impossible to find. We are aware of only one program for this purpose (Titan Technologies, 1982). It is probably not reasonable to assume that your end users will have available such cards, or the necessary software.

\section{Disk I/O}

Although disk $\mathrm{I} / \mathrm{O}$ on the Apple is faster than on many of its competitors, it is nonetheless cumbersome, particularly given that, because of the small RAM available, it is often necessary to store and recover data from the disk rather than hold them in RAM. If frequent disk access is necessary, it would be helpful to increase its efficiency. In addition, the capacity of the normal 5.25-in. DOS-formatted Apple disk is only 496 sectors (about 127,000 bytes). It is easy to exceed this limit if large data files are needed, or if both programs and data are to be stored on the same disk.

Programming technique. 1. Since DOS 3.3 resides in RAM, there are many ways to modify it for a variety of purposes. These include changing DOS commands, removing commands, augmenting commands, bypassing checks on input, and so on. Particularly useful for laboratory applications are modifications that allow the user to create "data" disks without DOS or a greeting program (freeing up 32 disk sectors for program and data storage) and to display the free sectors remaining on a disk. A book such as The DOStalk Scrapbook (Weishaar \& Kersey, 1985), as well as many Apple-oriented magazines (e.g., Nibble, CALL A.P.P.L.E., Etc.) provide details.

2. Sequential files are normally read and written from the beginning to the end of the file. If the file is short or it does not need to be accessed often during execution, a sequential file will work fine. For many applications, however, random access files are preferred over the more commonly used sequential files. Random access files assume that data stored in them will always be placed in records (lines) of the same length, and in the same format. The advantage of random access files is that any individual record can be recovered or written easily, without searching or rewriting the entire file. Storage of data from subjects, for example, or of particular stimuli, might be facilitated using random access files. 
3. Routines may be included to allow the user to identify appropriate program disks and/or data disks, if these are to be separate. With the increasing availability of highcapacity floppy disk drives and hard disks, it should not be assumed that every computer will have the appropriate controller in slot 6 , or that the volume number will be the default (0). The following routine allows relatively easy selection of new default values for a data disk. Values for slot, drive, and volume number may be given separately or together (e.g., "D2,V6" or "S7') and are displayed before continuing.

$100 S=6: D=1: V=0$

: REM DEFAULT SLOT, DRIVE, VOL NO

$110 Y \$=$ ",

$:$ DD $=$ "S" + STR\$ (S) + ",D"

$+\operatorname{STR} \$(D)+“, V ”+\operatorname{STR} \$(V)$

120 HOME: VTAB 8: PRINT SPC( 8)

“INSERT DATA DISK, THEN"

130

PRINT: PRINT SPC( 6)

“PRESS < RETURN > FOR "DD\$

: PRINT : PRINT SPC( 10)

“'OR ENTER S\#,D\#,V\#:";

140 GET X\$

: ON X\$ = CHR\$(13) AND Y\$

= " " GOTO 210

: ON X\$ = CHR\$ (13) GOTO 160

: IF X\$ = CHR\$ (8) THEN X\$ = “’”,

: IF LEN (Y\$) $>1$ THEN Y\$

$=$ LEFT\$ $(Y \$$, LEN $(Y \$)-1)$

: HTAB ( PEEK (36))

Y\$ = Y\$ + X\$: PRINT X\$; :GOTO 140

FOR I = 1 TO LEN (Y\$)

$: X \$=\operatorname{MID} \$(Y \$, I, 1)$

$: X=\operatorname{VAL}(\operatorname{MID} \$(Y \$, I+1)$

$:$ IF X\$ $=$ "S" THEN $S=X$

IF $\mathbf{X} \$=$ " $D$ " THEN $\mathbf{D}=\mathbf{X}$

IF $X \$=$ " $V$ " THEN $V=X$

NEXT I

: IF S $<1$ OR S $>7$ OR D

$<1$ OR D $>2$ THEN

PRINT:PRINT range error message : GOTO 100

GOTO 110

program continues
4. To check for the existence of a file on disk, use UNLOCK preceded by an ONERR GOTO rather than OPENREAD or VERIFY. OPEN will create a (possibly unwanted) file; VERIFY examines each byte in the entire file, a time-consuming procedure if the file is long.

Software utilities. 1 . There are several routines that speed up disk I/O for DOS 3.3, for example, Diversi-DOS (Basham, 1982), ProntoDOS (Weishaar, 1982), and others. The speed increases range from about 2-5 times normal DOS, depending on the type of file being $\mathrm{read} / \mathrm{written}$. In addition, most of these will optionally move DOS to the upper $16 \mathrm{~K}$ memory area.

2. ProDOS handles files much more quickly than does DOS 3.3; it matches or exceeds the speed of most DOS enhancement utilities, except for SAVEs and BSAVEs, on which it is slower. It is optimized for use with largecapacity storage devices, such as hard disks, but may also be useful on standard two-drive systems. In addition, it supports a wider variety of file types (including a VAR type which holds Applesoft variables), has several additional commands, and provides considerably more flexibility in file organization. We have some doubts about its utility for instructional programming, largely because ProDOS (along with BASIC.SYSTEM) occupies all of the upper $16 \mathrm{~K}$ of memory, plus the space used by DOS 3.3, and makes Applesoft programs run slightly slower than they do under DOS 3.3. Still, the enhancements can simplify operation and, in many cases, are powerful and useful.

Hardware. 1. It is now possible to buy a 10-MB hard disk for the Apple for under $\$ 700$, and a 20-MB hard disk for under $\$ 1000$. A hard disk can be very useful, particularly for program development, when multiple copies of programs are kept, or for storing very large data files. In addition, disk $\mathrm{I} / \mathrm{O}$ with a hard disk is much faster than with a floppy disk. Unfortunately, it is not reasonable to suppose that most end users will have hard disks available, so it would not be a good idea to develop commercial programs under that assumption.

2. Most RAM expansion cards are provided with software that allows their use as a "pseudo-disk," or RAMdisk. A 128K RAM card will hold the contents of an entire (normal) DOS 3.3 disk. This addition, although again not likely to be available to end users, is a valuable programming development tool, and is well worth the modest price of approximately $\$ 120$. This is particularly true for programs that depend heavily on disk $\mathrm{I} / \mathrm{O}$; the RAM-disk can save time as well as wear and tear on disk drives during debugging and testing. Unfortunately, the software implementing the RAM-disk operations is often not compatible with other program development tools, such as program line editors, and also may not work with some ampersand utilities.

\section{Error Trapping User Input}

Both new and experienced users make a variety of errors. These include entering inappropriate information, attempting to execute program segments out of sequence, 
using an inappropriate program or wrong disk, failing to follow or misinterpreting instructions provided by programs, and so on. In addition, even thoroughly tested programs may themselves contain errors. These errors should be anticipated and provided for. This is particularly important for commercial programs.

Programming technique. 1. Avoid the INPUT command. Applesoft has no equivalent of the LINPUT (line input) command found on many other computers. INPUT uses the "," and ":", as separators (for multiple-value inputs); information following these characters causes an EXTRA IGNORED message. INPUT does not accept strings containing a quote (it will give a ?REENTER message), and any blanks preceding the input string also are ignored. The results can be confusing.

2. Assign input to character strings, preferably using an "input anything" routine that can be examined for bad input. A simple routine, which is usable if relatively few entries are to be requested and time is not critical, is

$10 \mathrm{IL}=5:$ REM MAX LENGTH INPUT STRING

20 ST\$ = "”: REM INPUT STRING

30 GET X\$

$$
\begin{aligned}
& \text { : IF X } \$<>\text { CHR\$ (13) AND LEN (ST\$) } \\
& <255 \text { THEN ST\$ } \\
& =\operatorname{MID} \$(\text { ST\$, } 1, \text { LEN }(\text { ST\$) }-(\text { ASC }(X \$) \\
& =8) *(\operatorname{LEN}(\text { ST\$ })>0)) \\
& +\operatorname{MID} \$(X \$, 1+(\operatorname{ASC}(X \$)<32)) \\
& \text { : PRINT MID\$(X\$ + CHR\$ (7),1 } \\
& +(\text { ASC }(\mathrm{X} \$)<32 \text { OR LEN }(\mathrm{ST} \$)>\mathrm{IL}) \\
& \text { - ( } \operatorname{ASC}(X \$)=8), 1) \text {; } \\
& : \text { ST\$ = LEFT\$ (ST\$,IL) } \\
& \text { : GOTO } 30
\end{aligned}
$$

$\mathbf{N}=$ VAL (ST\$) : REM EVALUATE IF NEEDED

This routine sounds a "beep" for illegal characters or characters beyond the maximum length of IL. The strings can be converted to numerical values using the VAL command and then checked for range errors. Unfortunately, this approach is slow and can lead to problems with garbage collection if many strings are defined. In addition, it does not accept use of the forward arrow for input, although this could be included if desired.

If no checks on input are desired, a simpler routine (Weishaar \& Kersey, 1985) uses an Apple monitor routine at 64879 for this purpose and stores the input in the page 2 buffer, which can then be pulled into a string:

$50 \quad$ CALL -657

$$
\begin{aligned}
& : A \$=“ " \\
& : \text { FOR I = 512 TO } 767 \\
& : P=\text { PEEK(I) }
\end{aligned}
$$

$$
\begin{aligned}
& : \text { IF } \mathrm{P}<>141 \text { THEN } \\
& \text { A\$ }=\text { A } \$+\text { CHR } \$(P) \\
& : \text { NEXT I }
\end{aligned}
$$

A better alternative is to use one of several machine language routines available (e.g., Weishaar, 1985a; Mottola, 1982).

3. Errors should be trapped and handled using the ONERR GOTO and RESUME commands, but problems can occur if an ONERR is encountered in certain situations. A fix for these problems is given on page 136 of the Applesoft II BASIC Programming Reference Manual (Apple Computer, 1978). The virtue of ONERR GOTO is that it allows the program to examine the error that occurred. If the program is attempting to read or write a file, for example, and the user has left the drive door open, an I/O ERROR (error code \#8) is generated; a program searching for a nonexistent file will produce a FILE NOT FOUND (code \#6) error. The error code can be found by PEEKing at location 222; this value can be used to select an appropriate message to be printed. In some cases there may be nothing the user can do about the problem, so it is desirable to include a "fatal error" message as well. The error type (from the PEEK(222) command) and the line number in which the error occurred (found by PEEK(218) + PEEK(219) * 256) should also be printed if there is no remedy available to the user.

4. If desired, CONTROL-RESET can also be trapped (see Little, 1982: Weishaar, 1985c), although we have some doubts about the virtues of doing so in educational software, since users may want to interrupt the program during execution to examine variables or make changes.

Software utilities. There are software utilities that contain a variety of elaborate routines for I/O error trapping; the most comprehensive and suitable for instructional programming that we know of is CONDUIT's StringKit (CONDUIT, 1986). This software has routines for entering strings that contain commas and colons, converting strings to numeric values, evaluating the input for range and type errors, as well as formatting output for printing to the screen.

\section{Screen and Printer Displays}

Presentation of clear and concise displays to the screen is an important part of instructional programming. The major difficulties are: (1) presenting too much information, leading to screen "clutter," (2) presenting too little information (especially unlabeled information), (3) poor character presentation (e.g., overuse of inverse or flashing characters, marginally readable characters on the graphics screens), and (4) poor alignment (e.g., in columns and rows, or with varying numbers of decimal values). The first three of these have obvious solutions and are not discussed here.

Programming technique. 1. Use of the SPC( command to center and align material is generally easier and more general than $\mathrm{HTAB}$ pos or POKE 36, pos +1 , par- 
ticularly if the same routine is used for printing to the screen as well as to a printer. In addition, HTAB does not work with most printers, and POKE 36 will not work with 80-column boards. A small adjustment to the normally used centering routine

\section{PRINT SPC( 20 - LEN(ST\$)/2) ST\$}

allows strings to be centered on either the printer or the screen.

$10 \mathrm{EP}=1:$ REM eighty column card or printer active

$$
\text { (1=no, } 2=\text { yes })
$$

$$
20 \mathrm{~S}=40 * \text { EP }- \text { LEN }(\mathrm{ST} \$) / 2
$$

30 PRINT SPC $(S *(S>=0))$ ST\$

This checks to ensure that the string does not produce an ILLEGAL QUANTITY error if the string exceeds the available space.

Whole numbers (for numbering data points, stimuli, responses, etc.) can be easily aligned using

10 PRINT SPC $(\mathrm{N}<10) \mathrm{N}$;

or

10 PRINT SPC( $N<10) \operatorname{SPC}(N<100) N$;

2. Several PRINT USING routines are available to allow appropriate formatting of numbers in columns. A slight modification of a simple one provided by Crossley (1980) works fine for many applications:

50 REM X = number to be printed, $\mathrm{N}=$ no. decimal places, $S=$ width of field -1 , TN $=10$,

$\mathrm{PF}=.5, \mathrm{O}=1$, (one), $\mathrm{Z}=0$ (zero),

$\mathrm{B} 1 \$="$ ", $\mathrm{ZD} \$=$ " $0.00 "$

60 IF $N=Z$ THEN $X=$ INT $(X+P F)$

: PRINT SPC( $S+O-$ LEN ( STR\$ (X)))X;

: GOTO 130 : REM no decimal points

$70 \mathrm{X} \$=\mathrm{B} 1 \$+\mathrm{STR} \$($ INT $(\mathrm{X} * \mathrm{TN} \wedge \mathrm{N}+\mathrm{PF}))$

$80 \mathrm{Q}=\mathrm{LEN}(\mathrm{X} \$)-(\mathrm{VAL}(\mathrm{X} \$)<\mathrm{Z})$

$: \mathrm{Q} 1=(\mathrm{X}<\mathrm{O}$ AND $\mathrm{X}>-\mathrm{O})$

90

PRINT SPC $(S-Q *(Q>N+O)-(N+2)$

* $(\mathrm{Q}<=\mathrm{N}+\mathrm{O})+\mathrm{Q} 1)$;

100

$(\mathrm{Q}<=\mathrm{N})+(\mathrm{Q}-\mathrm{N}) *(\mathrm{Q}>\mathrm{N}))$;

PRINT MID\$ $(Z D \$, O+((N+O)<Q)+Q 1$,

$\mathrm{O}+(\mathrm{N}-\mathrm{Q}+2) *(\mathrm{Q}<\mathrm{N}+2)-\mathrm{Q} 1)$

120

$+(\mathrm{Q}-\mathrm{O}) *(\mathrm{Q}<=\mathrm{N}))$
More elaborate and faster machine language versions can be found elsewhere (e.g., Morris, 1980).

3 . It may help to include a routine to determine on which type of computer the software is running. Many institutions have several versions of the Apple, $\Pi+s$, Пes, and IIcs. Although the Пle and IIc have a built-in lowercase character generator ROM, the II+ prints in uppercase only; unless a special character generator ROM is added, lowercase will appear as garbage. Even with the addition of the character generator ROM and a shift key modification, special software is needed to INPUT lowercase characters on the II + . Golding (1985) indicates that the type of machine can be found in the following identifying bytes, which can be PEEKed by a running program:

\$FBB3 \$FBC0 \$FB1E

Apple II 38

Apple II+

EA

EA

$\mathrm{AD}$

Apple III (in emulation mode)

06 EA

Apple Ie (original)

Apple IIe (enhanced)

Apple IIc

$06 \quad$ E0

$06 \quad 00$

If the standard identification bytes $(\$ \mathrm{Cs} 05, \$ \mathrm{Cs} 07$, and $\$ C$ OB) are used, the following (Weishaar, 1985b) may check for various peripherals.

10 CARD $=$ INT $($ PEEK $(49164)+($ SLOT $* 256)) / 16$

20 IF CARD $=1$ THEN slot contains a printer

30 IF CARD $=2$ THEN slot contains a joystick or mouse

40 IF CARD $=3$ THEN slot contains a serial or parallel card

$50 \quad$ IF CARD $=4$ THEN slot contains a modem

60 IF CARD $=5$ THEN slot contains a sound or speech device

70 IF CARD $=6$ THEN slot contains a clock

80 IF CARD $=7$ THEN slot contains a disk or other storage device

90 IF CARD $=8$ THEN slot contains an 80-column card

100 IF CARD $=9$ THEN slot contains a network or bus interface

110 IF CARD $=10$ THEN slot contains none of the above

(Although this routine correctly detected our 80-column card and printer, it failed to detect an active 16K RAM card in slot 0 of an Apple II+, an inactive 128K RAM card in slot 3, or a Sider II in slot 7. We have not checked it with other peripherals.) 
4. On the Ile, using an 80-column card requires a memory card in the auxiliary slot (0). To check, this routine (Weishaar, 1985b) may be used:

210 EIGHTY $=1:$ REM ASSUMES 80 COLS

220 POKE 49153,0 : POKE 49237,0

\section{: REM AUXMEM SCREEN ON}

230 FOR $\mathrm{I}=0$ TO 255 STEP 8

240 POKE 1024, I

250 IF PEEK $(1024)<>$ I THEN EIGHTY $=0$

\section{: REM CHECK IF POKE STICKS IN MEM}

260 POKE 49236,0 : POKE 49152, 0:

\section{REM TURN AUXMEM OFF}

If there are several programs in the system, this information can be stored in a system configuration file at the beginning of each run, and then read in by the other programs.

5. Text files read from disk should be in mixed upperand lowercase format, taking advantage of the built-in upper/lowercase capabilities of the Ile and IIc. If software is being run on an Apple II+, one can determine whether it has a lowercase character generator ROM by printing a message in lowercase to the screen with some instructions that can be performed only if the message can be read:

\section{please type 'yes,' then}

\section{PRESS RETURN TO CONTINUE:}

If no lowercase character generator ROM is installed, the upper line will appear as garbage, and the program should convert lowercase characters to uppercase:

110 FOR I = 1 TO LEN( ST\$ )

: PRINT CHR\$( ASC( MID\$( ST\$, I, 1)

$-32 *(\operatorname{ASC}(\operatorname{MID} \$(\operatorname{ST} \$, I, 1)>95))))$;

\section{: NEXT I}

This could be better done in machine language, of course. The segment shown in Table 1 (from Huang, 1986) is typical.

6. A "HELP"' screen can be implemented fairly easily on the page 2 text screen. A simple technique for doing so can be found in "Tip Book \#7" (Beagle Bros., 1983).
7. If considerable data entry is needed, a simple function can be used to convert the letter keys on the Apple into a numeric keypad. Left-handed users should be given the option of a redefined keyboard.

100

$$
\begin{aligned}
& \text { DEF FN KP(X) }=1 \text { * (X\$ = “M”) } \\
& +2 *(X \$=“, ")+3 *(X \$=“ .,) \\
& +4 *(X \$=“ J ”)+5 *(X \$=“ K ”) \\
& +6 *(X \$=“ L ”)+7 *\left(X \$=“ U^{\prime \prime}\right) \\
& +8 *(X \$=\text { "I" })+9 *(X \$=“ O ”)
\end{aligned}
$$

Other keys must be defined as the zero and decimal point (e.g., SPACE BAR and "N"), and of course, the values should be checked for unacceptable keypresses.

Machine language versions of a numeric keypad are also available (see Cartaya, 1982; Cohen, 1983).

8. Users should be given the opportunity to back up to a previous page. This is not as easy as it sounds, since often calculations are performed or information is read from or written to the disk which prevent it. Careful program design can often minimize these situations. The "ESC" key is an obvious candidate for a "help," "backup," and "quit" function.

Software utilities. 1. Use of a word processor to create ASCII TEXT files to be read in by the program often allows cleaner and more readable screen displays and is easier than typing PRINT statements, especially if much information needs to be presented. Many word processors allow formatted text to be written to the disk, so appropriate page (screen) lengths and margins (including right justification, if desired) can be set. If such a word processing capability is not available, an alternative technique for formatting screen displays (which allows word wraparound only at word breaks) can be used (e.g., CONDUIT, 1986; Huang, 1986). Long user input should also be appropriately displayed. StringKit (CONDUIT, 1986) uses the graphics screen for all text printing and is probably too slow for timed displays.

2. Routines for opening and using Macintosh-like windows are given by Lew (1986); Seeds (1985) provides simpler versions.

\section{Miscellaneous}

Laboratory software often requires special techniques, such as randomization, sorting, and tone generation.

Programming techniques. 1. The Apple's RND( function does not generate "true" random numbers. In par-

Table 1

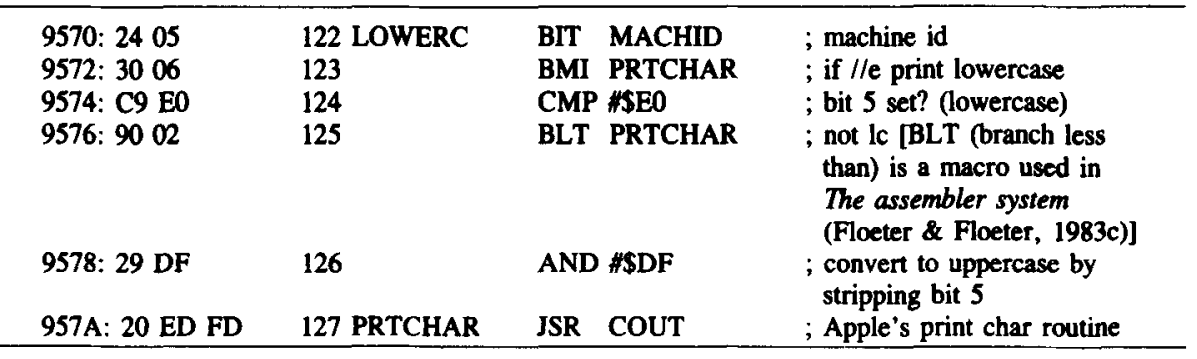


ticular, $\mathrm{RND}(1)$ generates the same sequence of numbers each time the computer is turned on. Obviously, this will not work for many laboratory applications, in which we hope to randomly permute the sequence of, say, stimuli to be presented for each subject. This can be corrected by using a different "seed" value the first time a random number generator is needed. A nearly random "seed" can be found at locations 78 and 79 , which are continuously updated while the computer waits for a keypress. Provided the user has had to press a key before the randomization routine is called, this can provide different "seeds." Other ways to provide random numbers are discussed by Root (1986).

2. Assuming that one has a "true" (or nearly true) way of getting a random number, a simple and fast way of permuting an array in BASIC is given by N. J. Castellan, Jr. (Personal communication, November 25, 1986):

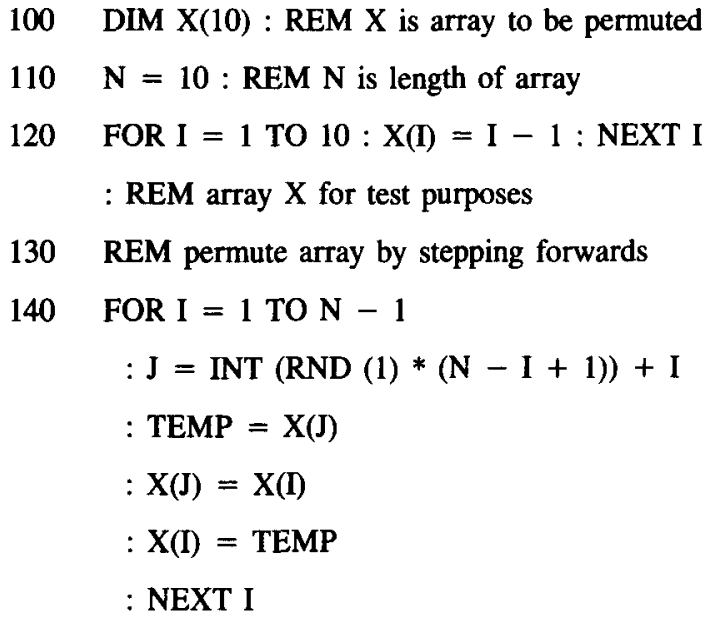

This works by swapping each element of the array for another element. Note that each item to be permuted is selected only once. It can be used with arrays containing any values or with string arrays, although pointers to strings should be used, rather than strings themselves. Castellan also observes that it is important in generating random numbers that the random sequence generator not be reseeded with each call.

3. For most instructional laboratory applications, esoteric sorting routines are probably not necessary. If exceptional speed is needed, machine language routines are available (see Kiziak, 1984).

4. Tones can be easily generated on the Apple's speaker, but one must be cautious how they are used, since the same routine may produce audibly different pitches on different machines. The tones produced may also be affected by the presence of peripheral cards, especially 80 -column boards. A tone generation routine can be found in De Jong (1982).

5. Programs that use the graphics screens (or the page 2 text screen) may cause problems. The normal start of an Applesoft program is at the beginning of the page 2 text screen; longer programs may overlap the graphics screens, which are located in the middle of the RAM available for program storage. A routine for moving a program which uses the graphics screens or text page 2 is easy to implement for moderate length programs:

$$
10
$$

$$
\begin{aligned}
\mathrm{PG}= & 64: \text { REM page to move above } \\
& (8=\text { normal start of program, } \\
& 12=\text { above text page } 2,64=\text { above }
\end{aligned}
$$

hi-res page $1,96=$ above hi-res

page 2)

$$
\text { ST }=\text { PG * } 256: \text { REM start of program }
$$

30 IF PEEK (103) $<>1$ OR PEEK (104)

$$
\begin{aligned}
& <>\text { PG OR PEEK (ST) } \\
& <>0 \text { THEN POKE 103, } 1
\end{aligned}
$$

: POKE 104, PG

: POKE ST, 0

: PRINT

: PRINT CHR\$(4) "RUN programname"

For programs that also need the space below the hi-res pages, program "splitters" may be used. These use the $6 \mathrm{~K}$ available below the hi-res pages, as well as the memory above them (see James, 1984).

\section{Getting Around BASIC as a Language}

Applesoft BASIC lacks many desirable features of a more powerful structured BASIC, including long variable names, PRINT USING, simple disk access commands, and so on. In addition, control structures, such as IFTHEN-ELSE, DO-UNTIL, WHILE-WEND, and CASE$O F$, are absent, encouraging unrestricted use of the GOTO. Programmers may find other languages, such as Pascal, C, or FORTH, more suitable and appropriate for many applications. Nonetheless, Applesoft BASIC's availability as the resident language in the Apple II makes it probably the most well known and used language for programming in student laboratory applications.

Programming technique. 1. In spite of its drawbacks, it is possible to write structured programs in Applesoft. Advocates of structured programming argue for modularity, top-down design and testing, and use of simple control structures: sequencing, selection, repetition, and subroutines. Modularity can be implemented by ensuring that each portion of the program perform only one easily identifiable function and have only one entry and exit point. Applesoft places no roadblocks to top-down design and testing. It is possible to use Applesoft's FORNEXT capability, given appropriate tests, to simulate structured programming control structures (see Peterson, 1984).

2. Use of a word processor to create programs can be helpful. Programs can be indented appropriately for IF 
statements and loops. Some word processors allow standard ASCII text files to be stored on disk with line numbers even when no line numbers are present in the file itself; if a user's word processor allows this, the text file can simply be EXECuted into RAM as a BASIC program.

3. Frequently used subroutines should be saved in a subroutine library, preferably with line numbers different from those likely to be used in your program. These can be EXECuted into the program and renumbered appropriately.

Software utilities. There are many utilities designed to facilitate programming in Applesoft. These include BASIC line editors, programs to compress programs, preprocessors that facilitate structured programming, compilers, and other aids.

1. Other than a renumber/merge capability, the most valuable aid to programming we have found is a program list formatter that prints each statement on a single line, indents the THEN portion of IF-THEN statements, and indents loops. XLister (Kersey, 1981) is one such program.

2. BASIC line editors such as GPLE (Konzin, 1982) or G.A.L.E. (Mossberg, 1983) can be very helpful, although we know of none that provides full line editing capabilities as well as a renumber/merge capability, listing of variables, variable values, cross reference, and other program development tools.

3. The best Applesoft-based programming environment we know of is Blankenship BASIC (Blankenship, 1984). This interpreted structured BASIC (implemented by use of hidden ampersand commands) is compatible with Diversi-DOS (Basham, 1982) and a RAM disk. It contains all of the standard structured BASIC commands, as well as many other useful routines missing in Applesoft (e.g., PRINT USING, SORT, SWAP, INKEY, INLINE, BOX, BOXFILL, INSTR\$, selective DELETE ARRAY, fast garbage collection, and many others). It also has its own editor, a LIST command that calls out IF-THENs and loops, and a built-in renumbering command (although we believe some improvements could be made in these). Blankenship also has compatible programs that provide memory optimization by compressing lines and removing REMs (Blankenship, 1985b) and a character editor (Blankenship, 1985a).

4. "Preprocessors,"' which provide structured control procedures, GOTO named subroutines, and so forth, might be helpful under some circumstances. Unfortunately, they are often cumbersome to use; after entering the program using the structured BASIC commands, it must be converted to "standard" Applesoft using the preprocessor before it can be run. Programmers accustomed to interactive testing during program development will find this annoying. Furthermore, although the initial coding looks nice, after preprocessing the result is often a jungle of GOTOs. A better alternative is a structured BASIC compiler, such as ZBASIC (Gariepy et al., 1985) or Micol BASIC (Micol Systems, 1985). ZBASIC runs only under DOS 3.3, and requires an Apple IIe for program development (although the programs will run on a II +); Micol BASIC runs only under ProDOS.

5. A critical part of program development is testing and debugging. Williams (1985) provides a short but thorough discussion of the use of PRINT statements, breakpoints, snapshots, and the need for separate debugging and testing phases. A "Trap and Step"' facility (Floeter, 1982) can be very handy for these purposes, as well as a subroutine for printing selected values of variables during run time. A branch to a routine that prints the error code and line number in which the error occurred can also be helpful.

Hardware. 1. One of the most helpful hardware additions that can be made for program development is a goodsized RAM card or extended 80-column board, which can be used during program development as a pseudo-disk for testing disk-intensive programs. Another useful card is an accelerator, which can speed up testing of programs, although it should not be expected that end users will have these available.

2. An excellent tool for documentation is an automatic text-to-printer dump card, which allows printing of text and screen graphics without having to modify the program code for this purpose. Print-it (by Textprint) is one such card.

\section{SUMMARY}

Although programming on the Apple can be difficult and at times exasperating, it is possible to write excellent programs for the student laboratory if some effort is made to take advantage of procedures such as those mentioned above. We have only touched on the immense literature available; for each routine discussed, there are dozens more that perform related functions. Magazines and newsletters, such as Nibble, Call A.P.P.L.E., Apple Assembly Lines, Byte, Open-Apple, inCider, and others, are filled with helpful material. In addition, back issues of the now defunct Micro, Kilobaud Microcomputing, Creative Computing, and Softalk are also excellent sources of information. Some sources publish annual indexed collections of articles that have appeared in their pages (e.g., Nibble, Open-Apple), which are preferred to the original articles, since bugs are usually corrected and updates are included. Nibble supplies many of the routines and utilities it publishes in collections on disk for reasonable prices. Collegiate Microcomputer often contains useful suggestions for using computers in classroom and laboratory settings. Behavior Research Methods, Instruments, \& Computers regularly publishes generalizable programs and algorithms for the Apple and other computers.

\section{REFERENCES}

APPLE COMPUTER, INC. (1978). Applesoft II BASIC programming reference manual. Curpertino, CA: Author.

APPLE COMPUTER, INC. (1980a). Applesoft tool kit [Computer program] Cupertino, CA: Author

APPle CoMPuter, INC. (1980b). CHAIN [Computer program]. Cuper- 
tino, CA: Author.

Basham, B. (1982). Diversi-DOS [Computer program]. Farmington, MI: Diversified Software Research.

Basham, B. (1985, March). Basham fires up the incinerator [Letter to the editor]. Open-Apple, 1, 17-19.

BeAGle Bros. (1983). Tip book \#7. In N. Konzen, GPLE: Global program line editor [Computer program manual]. San Diego, CA: Author.

BlankenshIP, J. (1984). Blankenship BASIC [Computer program]. Atlanta, GA: Author.

Blankenship, J. (1985a). Character/Editor [Computer program]. Atlanta, GA: Author.

BlANKENSHIP, J. (1985b). Shrink/Expand [Computer program]. Atlanta, GA: Author.

BONGERS, C. (1982a). Amper PRINT-USE program. In Nibble express (Vol. 2, pp. 163-169). Lincoln, MA: MicroSPARC.

Bongers, C. (1982b). Amper store and recall. In Nibble express (Vol. 2, pp. 121, 136, 148). Lincoln, MA: MicroSPARC.

Bongers, C. (1983). Quick sort. In Nibble express (Vol. 3, pp. 9093). Lincoln, MA: MicroSPARC.

CarTAYA, R. (1982). The phantom numeric keypad! In Nibble express (Vol. 2, pp. 71, 83, 99). Lincoln, MA: MicroSPARC.

Castellan, N. J., JR. (1983). Strategies for instructional computing. Behavior Research Methods \& Instrumentation, 15, 270-279.

Conen, A. (1983). Super numeric keypad. Nibble express (Vol. 3, pp. 128-129). Lincoln, MA: MicroSPARC.

CONDUIT. (1986). StringKit. Iowa City, IA: Author.

ConNolly, R. (1981). Improving the multiple array sort. In Nibble express (Vol. 1, pp. 148-149). Lincoln, MA: MicroSPARC.

Crossley, J. (1980, March). Print using simulator. International Apple Core Application Notes (p. B8). Daley City, CA: International Apple Core.

DE JONG, M. (1982). Apple II assembly language. Indianapolis, IN: Sams.

Devine, R. R. (1986, June). The BASIC-assembly connection. Nibble, 86-91.

EAMON, D. B., \& BuTLER, D. L. (1985). Instructional programs for psychology: A review and analysis. Behavior Research Methods, Instruments, \& Computers, 17, 345-351.

FINDLEY, R. (1979). 6502 Software gourmet guide \& cookbook. Elmwood, CT: Scelbi.

Floeter, A. (1981). Applesoft REM remover. In Nibble express (Vol. 1, pp. 72-75). Lincoln, MA: MicroSPARC.

Floeter, A. (1982). Apple trap/step. In Nibble express (Vol. 2, pp. 101105). Lincoln, MA: MicroSPARC.

Floeter, A., \& Floeter, V. (1983a). Applesoft line cruncher. In Nibble express (Vol. 3, pp. 17-22). Lincoln, MA: MicroSPARC.

Floeter, A., \& Floeter, V. (1983b). Applesoft variable cruncher. In Nibble express (Vol. 3, pp. 77-81). Lincoln, MA: MicroSPARC.

Floeter, A., \& FLETER, V. (1983c). The assembler system [Computer program]. Concord, MA: MicroSPARC.

FLoeter, A., \& FloETER, V. (1983d). MacroSoft [Computer program]. Concord, MA: MicroSPARC.

GariePY, A., OVERTon, D., \& TeRry, S. (1985). ZBASIC [Computer program]. Tucson, AZ: Zedcor.

GoldING, V. (1985, May). Mousetext apologist surfaces [Letter to the editor]. Open-Apple, 1, 40.

GoRDON, B., FIELD, B. (1985). Screen blanking on the Apple Ile. Behavior Research Methods, Instruments, \& Computers, 17, 518.

Huang, H. (1986, July). Amperformatter. Nibble, 100-105.

IRWIN, P. (1982). Amper jump \& Tsort. In Nibble express (Vol. 2, pp. 141-147). Lincoln, MA: MicroSPARC.

IRWIN, P. (1983). Amp-L-Soft utilities. In Nibble express (Vol. 3, pp. 141-153). Lincoln, MA: MicroSPARC.

JAMES, G. (1984). Amper fre splitter. In Nibble express (Vol. 4, pp. 124 130). Lincoln, MA: MicroSPARC.

Kersey, B. (1981). XLister [Computer program]. San Diego, CA: Beagle
Bros.

KIzIAK, G. (1984). The compact sorter. In Nibble express (Vol. 4, pp. 25-29). Lincoln, MA: MicroSPARC.

KonZIN, N. (1982). GPLE; Global program line editor [Computer program]. San Diego, CA: Beagle Bros.

LEVENTHAL, L. (1979). 6502 assembly language programming. Berkeley, CA: Osborne/McGraw-Hill.

LEw, D. (1986, July). Window show. In Nibble, 56-71.

LitTle, G. (1982). Trapping the reset key. In Nibble express (Vol. 2, pp. 117-118). Lincoln, MA: MicroSPARC.

MiCol Systems. (1985). Micol BASIC. Toronto, Ontario, Canada: Author.

Mitchell, E. (1982). Searching techniques: Part 4. Creative Computing, 8, 284-294.

MoRris, G. (1980, October). Print using for Applesoft. Micro, 14-17.

Mossberg, S. (1983). G.A.L.E.: Global program line editor [Computer program]. Concord, MA: MicroSPARC.

Motrola, R. (1981a). Amper-interpreter. In Nibble express (Vol. 1, pp. 117-126, 128). Lincoln, MA: MicroSPARC.

Motrola, R. (1981b). Passing variables in Applesoft BASIC. In Nibble express (Vol. 1, pp. 113-115). Lincoln, MA: MicroSPARC.

MotToLA, R. (1982). Inputting strings with commas. In Nibble express (Vol. 2, pp. 69-71). Lincoln, MA: MicroSPARC.

Mueller, K., Gruenenfelder, T., Robertson, K., \& Carrell, T. (1982, April). Apple II interfacing for the novice. Paper presented at the meeting of the Eastern Psychological Association, Baltimore.

Perera, T. (1987). Writing and publishing instructional and research software. Behavior Research Methods, Instruments, \& Computers, 19, 84-87.

Peterson, C. (1984). Applesoft tricks. In Nibble express (Vol. 4, pp. 158-159). Lincoln, MA: MicroSPARC.

PRICE, J. M. (1979). Software timing for 6500 series microcomputers. Behavior Research Methods \& Instrumentation, 11, 568-571.

PRICE, J. M. (1986). Relocating 65xx software timing routines. Behavior Research Methods, Instruments, \& Computers, 18, 68-69.

ReED, A. V. (1979). Microcomputer display timing: Problems and solutions. Behavior Research Methods \& Instrumentation, 11, 572-576.

REYNolds, W. (1983). Amper-speed: Fast read and write for text files. In Nibble express (Vol. 3, pp. 63-66). Lincoln, MA: MicroSPARC. Root, J. (1986, April/May). Dice simulator. II Computing, 42-46.

SeEds, M. (1985, January). Applesoft windows. Nibble, 78-81.

SOFTWARE MASTERS, (1984a). The visible computer: 6502 [Computer program]. Bryan, TX: Author.

SOFTWARE MASTERS. (1984b). The visible computer: 6502 [Computer program manual]. Bryan, TX: Author.

Titan Technologies. (1982). EMBER [Computer program]. Ann Arbor, MI: Author.

WADDELL, D. (1984). A fast GOTO processor for Applesoft. In Nibble express (Vol. 4, pp. 18-23). Lincoln, MA: MicroSPARC.

WAGNER, R. (1982a). Assembly lines: The book. North Hollywood, CA: Softalk.

WAGNER, R. (1982b, November). Everyone's guide to assembly language, Part 26. Softalk, 114-124.

WAGNER, R. (1982c, December). Everyone's guide to assembly language, Part 27. Softalk, 77-82.

WeishaAR, T. (1982). Pronto-DOS [Computer program]. San Diego,

CA: Beagle Bros.

WeISHAAR, T. (1985a). Input absolutely anything. Open-Apple, 1, 6768,74 .

WeishaAr, T. (1985b). Pokes galore. Open-Apple, 1, 101.

WeishaAR, T. (1985c). Trapping reset. Open-Apple, 1, 16.

WeISHAAR, T., \& KeRSEY, B. (1985). The DOSTalk scrapbook. Blue Ridge Summit, PA: Tab Books.

Williams, G. (1985, June). Debugging techniques. Byte, 279-290.

Zimmerman, S. (1986, September). The real thing: Floating point mathematics. Nibble, 74-91. 